\title{
Orientierende Untersuchungen zum Einsatz von Klassierprozessen in der Nichteisenmetallurgie
}

\author{
Stefan Patscheider ${ }^{1}$, Helmut Flachberger ${ }^{1}$ und Jürgen Antrekowitsch ${ }^{2}$ \\ ${ }^{1}$ Lehrstuhl für Aufbereitung und Veredlung, Montanuniversität Leoben, Leoben, Österreich \\ ${ }^{2}$ Lehrstuhl für Nichteisenmetallurgie, Montanuniversität Leoben, Leoben, Österreich
}

Eingegangen 6. Juli 2017; angenommen 8. Juli 2017; online publiziert 20. Juli 2017

\begin{abstract}
Zusammenfassung: Die Trennung von polydispersen Körnerkollektiven nach Unterschieden in der Korngröße bzw. der Gleichfälligkeit wird in der Mineralaufbereitung als Klassierung bezeichnet. Die Anwendungsfälle wie auch die dafür zur Verfügung stehenden Aggregate und möglichen Verschaltungsvarianten sind ausgesprochen vielfältig.

Eine Sonderform der Klassierung ergibt sich dann, wenn die Trennung nach der Korngröße bzw. der Gleichfälligkeit von einem Sortiereffekt begleitet bzw. überlagert wird. Ohne Anspruch auf Vollständigkeit wird dies etwa dann möglich, wenn sich Unterschiede an Wertstoffgehalten in den verschiedenen Korngrößen- bzw. Gleichfälligkeitsklassen ergeben, etwa bedingt durch unterschiedliche Bildungsprozesse der Mineral-/Metall-Phasen oder auch durch selektives Zerkleinerungsverhalten der gesteinsbildenden Mineralphasen.

Im Rahmen der Dissertation des Erstautors durchgeführte orientierende Untersuchungen haben ergeben, dass Klassierprozesse auch in der Nichteisenmetallurgie für die kontrollierte An- oder Abreicherung bestimmter Elemente bzw. Phasen eingesetzt werden können. In Hinsicht auf die Aufarbeitung von Zwischenprodukten und die Wiederverwertung von Reststoffen aus der Metallurgie ergeben sich durch die Klassierung neue Möglichkeiten der Wertstoffgewinnung beziehungsweise Schadstoffausschleusung aus einer Prozesskette. Anhand dreier Beispiele sollen die diesbezüglichen Potentiale aufgezeigt werden.
\end{abstract}

Schlüsselwörter: Klassierung, Selektive Zerkleinerung, Anodenschlamm, Flugstaub, Bleischlicker

Dipl.-Ing. S. Patscheider ( $\square)$

Lehrstuhl für Aufbereitung und Veredlung,

Montanuniversität Leoben,

Franz-Josef-Str. 18,

8700 Leoben, Österreich

stefan.patscheider@unileoben.ac.at
Preliminary Investigations Regarding the Application of Screening and Classification in Non-Ferrous Metallurgy

Abstract: The separation of polydisperse particle systems according to the differences in particle size or settling velocity is referred to the mineral processing industry as screening or as classification, respectively. Manifold applications, process designs and equipment are available in this context.

A special form of screening- and classification is incidental, when the process is accompanied or superimposed by a separation effect. With no claim to completeness variations of metal grade in different particle-size or settlingvelocity classes are present due to differential genesis processes of mineral-/metal phases or to selective comminution behaviour of rock-forming minerals.

As part of a preliminary study in context with the doctoral thesis of the main author, screening and classification were tested as a process for enrichment or depletion of certain elements or phases with three samples from non-ferrous metallurgical industry. First results have shown that in regard to re-processing of intermediate and waste products from non-ferrous metallurgy, screening and classifying opens new possibilities with respect to their recycling methods, which are presented hereafter.

Keywords: Screening, Classifying, Selective comminution, Anode slime, Dust, Copper dross

\section{Einleitung}

Die Klassierung ist ein aufbereitungstechnischer Grundprozess, der in der Aufbereitung von primären Rohstoffen und sekundären Reststoffen vielfältige Anwendungen findet. Unter Klassierung wird im Allgemeinen die Trennung eines polydispersen Körnerkollektivs in unterschiedliche Korngrößen- bzw. Gleichfälligkeitsklassen verstanden, wobei die Einteilung nach Siebmaschenweiten mit definierten Querschnittsflächen oder über Gleichfälligkeitsklassen in 


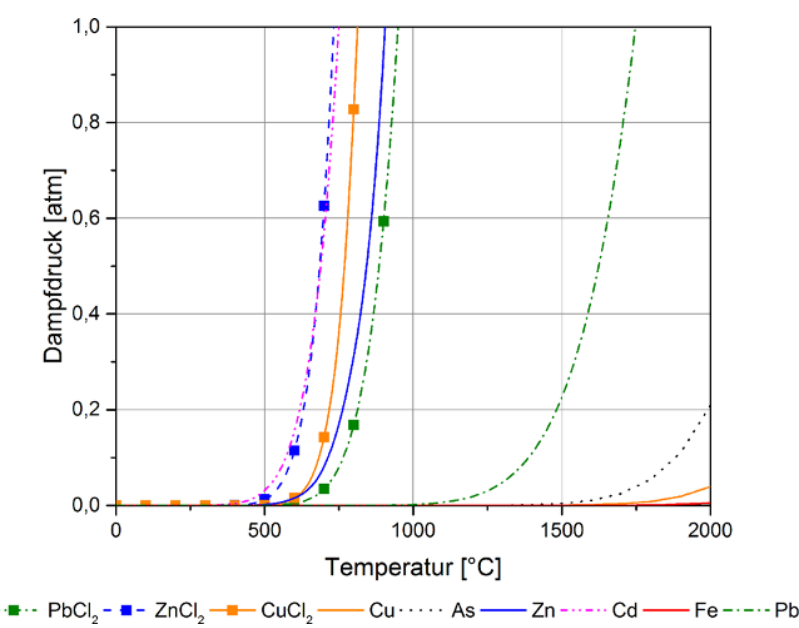

Abb. 1: Dampfdruckkurven von ausgewählten Metallen, Sulfiden und Metallhalogeniden

einem Fluidstrom erfolgt [1]. Klassierprozesse dienen u. a. zur Vorbereitung auf einen nachgeschalteten Zerkleinerungs- oder Sortierprozess oder der Einhaltung bestimmter Korngrößenverteilungen von Zwischenfraktionen bzw. Endprodukten.

In einigen Sonderfällen kann die Klassierung auch eine sortierende Wirkung entfalten, die für die gezielte Trennung nach Unterschieden in den Stoffeigenschaften genutzt werden kann. So sind bei der Aufbereitung von Industriemineralen einige diesbezügliche Beispiele bekannt: Etwa in der Kaolinaufbereitung, wo - vereinfacht ausgedrückt eine durch Verwitterungsprozesse entstandene Dispersitätslücke zwischen den sich im Feinsten anreichernden, vereinzelten Kaolinit-Lamellen und den sich im Gröberen anreichernden Quarzkörnern genutzt wird. Durch die Verwendung von geeigneten Klassierapparaten, wie Aufstromklassierern und Hydrozyklonen, können damit hochwertige Kaolinkonzentrate erzeugt werden [2]. Oder in der Aufbereitung von Chromiterzen, wo sich, bedingt durch unterschiedliche Bildungsprozesse, Unterschiede im selektiven Zerkleinerungsverhalten zwischen Chromit und den Begleitmineralen ergeben. Diese Mineralphasen reichern sich im Zuge der Zerkleinerung in verschiedenen Kornklassen an und können daher wiederum mittels eines Klassierschrittes in unterschiedlichen Korngrößenklassen aufkonzentriert werden.

Wie Untersuchungen des Erstautors zeigen, liegen auch in der Nichteisenmetallurgie, im Speziellen in der Kupferund Bleimetallurgie, ähnliche Potentiale vor, die einen Einsatz von Klassierprozessen in den etablierten metallurgischen Verfahrensroutinen als sinnvoll erscheinen lassen und in den nachfolgenden drei Beispielen kurz umrissen werden sollen.

\section{Beispiele aus der Nichteisenmetallurgie}

\subsection{Flugstaub aus einer Kupferhütte}

Bei pyrometallurgischen Prozessen entstehen neben der gewünschten Metallschmelze in der Regel auch Schlacke, mit der die unerwünschten Begleitelemente ausgeschieden werden, und Flugstaub, welcher mit dem Abgasstrom aus dem Prozessraum ausgetragen wird. Der Flugstaub setzt sich üblicherweise aus chemisch-physikalisch gebildeten und aus mechanisch mitgerissenen Fest-/Flüssig-Phasen zusammen [3]. Je nach Schmelzofentyp können z. B. bis zu $10 \%$ der Einsatzmenge im Flugstaub ausgetragen werden [4]. Aufgrund der hohen Wertmetallkomponente wird der Flugstaub beispielsweise in der Kupfermetallurgie für die Kupferrückgewinnung zurück in den Schmelzofen geführt, womit allerdings eine Reduzierung der Ofenkapazität einhergeht. Daher wurden diverse hydrometallurgische Aufarbeitungsverfahren für unterschiedliche Arten von Flugstäuben entwickelt.

Der mechanisch mitgerissene Partikelschwarm setzt sich aus den Einsatzstoffen, Schlackepartikeln und - je nach Ofentypus - auch aus Schmelzpartikeln, die durch Ausgasungen aus der Schmelzphase entstanden sind, zusammen. Chemisch-physikalisch gebildete Partikel bilden sich durch das Verdampfen bestimmter Elemente und Verbindungen wie beispielsweise Arsen und Cadmium, die im Abgassystem durch die Reduktion der Abgastemperatur resublimieren.

An den beiden an der Montanuniversität Leoben beheimateten Lehrstühlen für Aufbereitung und Veredlung sowie für Nichteisenmetallurgie wurden in den letzten Jahren gemeinsam mit einer Reihe von Industriepartnern diverse Flugstäube aus der Stahl- und Nichteisenmetallurgie auf Aufbereitbarkeit untersucht. Verwiesen sei hierbei etwa auf die Dissertation von Öfner 2013, deren wesentliche Erkenntnisse in [5] vorgestellt werden. Die Ergebnisse dieser Untersuchungen zeigten, dass sich die Anteile an mechanisch mitgerissenen und chemisch-physikalisch gebildeten Partikeln über verschiedene Partikelgrößenklassen hinweg unterscheiden. Diese grundlegende Erkenntnis eröffnet neue Wege in der Aufarbeitung von Flugstäuben aus metallurgischen Prozessen.

Gegenstand einer dieser Untersuchungen ist der Flugstaub einer Kupferhütte, welche vermehrt diverse Elektronikschrotte im pyrometallurgischen Prozess einsetzt. Durch den Einsatz dieser Schrotte gelangen die in manchen Kunststoffen enthaltenen Halogenverbindungen in den Schmelzbereich [6]. Metallhalogenide weisen eine im Vergleich zu Metallen deutlich niedrigere Verdampfungstemperatur auf. Auf Basis thermodynamischer Daten kann der Dampfdruck von Metallen, Sulfiden und Halogeniden berechnet werden. Wie aus Abb. 1 hervorgeht, verdampfen die aufgelisteten Halogenide, Cadmium und Zink unter $906^{\circ} \mathrm{C}$, was darauf schließen lässt, dass die im Flugstaub zu findenden Halogenide chemisch-physikalisch gebildet wurden. Die Kurven basieren auf Berechnungen mit HSC Chemistry 7.

Auf Basis dieser Erkenntnis sollten somit eine Anreicherung der Schadstoffkomponenten im Feinkornbereich und eine Anreicherung der Wertstoffkomponente Kupfer, das 


\begin{tabular}{|c|c|c|c|c|c|c|}
\hline $\mathrm{Cu}$ & $\mathrm{Fe}$ & $\mathrm{Pb}$ & $\mathrm{Zn}$ & $\mathrm{Cl}$ & $F$ & $\mathrm{Br}$ \\
\hline 2,7 & 0,7 & 12,4 & 43,3 & 4,8 & 1,3 & 3,5 \\
\hline
\end{tabular}

bevorzugt mechanisch mitgerissen wird, im Grobkornbereich möglich sein. Für die Untersuchung stand ein Flugstaub aus einem Badschmelzofen einer sekundären Kupferhütte zur Verfügung. Dieser Flugstaub weist üblicherweise hohe Zink-Gehalte auf und wird zur Aufarbeitung verkauft. Aus der chemischen Analyse des Flugstaubes (Tab. 1) ist ersichtlich, dass - neben Zink und den Schadstoffkomponenten - auch ein gewisser Anteil des Kupfers teilweise für die Hütte verloren geht. Mit der Klassierung des Flugstaubes sollte eine Reduzierung des Kupferverlustes möglich sein. Die Klassierversuche wurden mit dem am Lehrstuhl für Aufbereitung und Veredlung zur Verfügung stehenden Kanalradsichter Hosokawa Alpine 100 MZR durchgeführt.

Die Ergebnisse der Untersuchungen des Flugstaubes aus der sekundären Kupferhütte mit dem Kanalradsichter sind in Abb. 2 in Form zweier Henry-/Reinhardt-Schaubilder dargestellt. Durch die Klassierung im Fliehkraftfeld konnte der Kupfergehalt von 2,7\% in der Aufgabe auf 8,8\% in der Grobkornfraktion, bei einer nominellen Trennschnittlage von $25 \mu \mathrm{m}$ (siehe erste Klasse von oben in Abb. 2), angereichert werden. Mit dieser Fraktion können bei einem Masseausbringen von 8,4\% immerhin 27,1\% des in der Aufgabe enthaltenen Kupfers ausgebracht werden. Zugleich können die Elemente Zink, Blei, Fluor, Chlor und Brom in dieser gröbsten Partikelfraktion abgereichert werden. In der Feinfraktion konnte der Zinkgehalt von ursprünglich $43,3 \%$ in der Aufgabe auf 46,3\% geringfügig erhöht werden. Durch die veränderte chemische Zusammensetzung der Klassierprodukte gegenüber dem Aufgabegut ergeben sich neue Potentiale der Aufarbeitung. So könnte beispielshalber durch diesen Prozessschritt das Grobgut mit der erhöhten Wertmetallkomponente Kupfer zurück in den Schmelzofen geführt werden, um den Verlust an Kupfer für die Hütte zu reduzieren. Weiterführende Versuche zur Optimierung des Verfahrens sind derzeit Gegenstand fortgesetzter Forschungsaktivitäten der beiden vorgenannten Lehrstühle.

\subsection{Anodenschlamm aus der Kupferelektrolyse}

In der metallurgischen Verarbeitung von Kupfererzkonzentraten zu hochreinem Kupfer stellt die elektrolytische Raffination einen wesentlichen Prozessschritt dar. Dabei werden Kupferanoden mit einem Kupfergehalt von >99,5\% in Schwefelsäure gelöst und an Kathoden mit einem Kupfergehalt von $>99,99 \%$ abgeschieden. Ein ökonomisch wichtiges Nebenprodukt, welches bei diesem Prozessschritt anfällt, ist der sogenannte Anodenschlamm. Seine Zusammensetzung ist abhängig von den Ofeneinsatzstoffen, meist weist dieser jedoch hohe Gehalte an Silber, Gold und anderen Edelmetallen auf. Ein weiteres Element, welches auch im Anodenschlamm vorzufinden ist, ist Barium, da bei der Herstellung der Anodenformen Baryt als Schlichte eingesetzt wird. Die dabei zum Einsatz gebrachte Barytsuspension soll die Anodenform reinigen und ein schnelles Lösen der Anode von der Form ermöglichen [7]. Die Verwendung von Baryt wird hierbei bevorzugt, da sich dieser im Elektrolysebad inert verhält und keine Verunreinigung des Kathodenkupfers bewirkt.

Ein häufig eingesetztes Verfahren zur Aufarbeitung dieser Anodenschlämme ist die schwefelsaure Laugung zur Reduktion des Kupfergehaltes und die anschließende pyrometallurgische Weiterverarbeitung des Laugungsrückstandes zu Rohsilber. Für die Verarbeitung des Anodenschlammes wurden auch einige hydrometallurgische Verfahren entwickelt.

Am Lehrstuhl für Aufbereitung und Veredlung wurden Anodenschlammproben einer Kupferelektrolyseanlage untersucht, in Tab. 2 ist die chemische Analyse der Eingangsprobe dargestellt.

Die Aufarbeitung des untersuchten Anodenschlamms erfolgt durch eine schwefelsaure Laugung mit anschließender pyrometallurgischer Aufarbeitung des Laugungsrück-
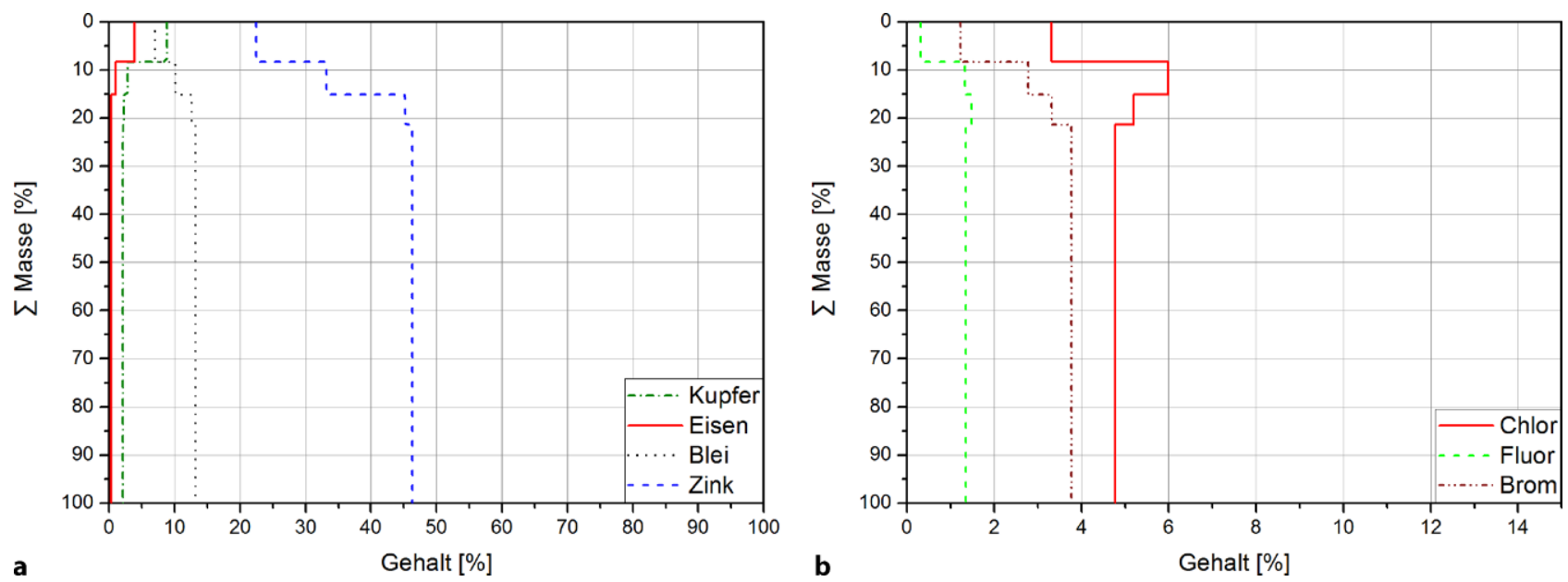

Abb. 2: a und b Henry-/Reinhardt-Schaubilder für den untersuchten Flugstaub nach den Sichtversuchen. Die Partikelgröße nimmt von oben nach unten $a b$ 


\begin{tabular}{|l}
\begin{tabular}{|lllllll|}
\hline $\begin{array}{l}\text { TABELLE } 2 \\
\text { Gehalte aus der chemischen Analyse eines unter- } \\
\text { suchten Anodenschlammes aus einer Kupferelektro- } \\
\text { lyse, } \mathbf{m} \%\end{array}$ \\
\hline $\mathrm{Cu}$ & $\mathrm{Pb}$ & $\mathrm{Sb}$ & $\mathrm{Sn}$ & $\mathrm{Ag}$ & $\mathrm{Au}$ & $\mathrm{Ba}$ \\
\hline 3,92 & 21,71 & 9,45 & 6,76 & 7,38 & 0,27 & 13,16 \\
\hline
\end{tabular}
\end{tabular}

standes in einem Treibkonverter (TBRC), in welchem als Endprodukt Rohsilber erzeugt wird. Der hier untersuchte Anodenschlamm weist einen erhöhten Barium-Gehalt auf. Nun führt der enthaltene Baryt bei der pyrometallurgischen Verarbeitung zu einer Erhöhung der Viskosität der erzeugten Einschmelzschlacke. Die erhöhte Viskosität der Einschmelzschlacke wiederum bewirkt ein vermindertes Fließverhalten ebendieser, was zu einer schlechteren Trennung von der Metallschmelze führt. Da in der Einschmelzschlacke noch beträchtliche Gehalte an Wertmetallen vorliegen, wird diese in einem nachgeschalteten pyrometallurgischen Prozess weiterverarbeitet, in dem der enthaltene Baryt die Schlackenbildung abermals beeinträchtigt. Für die stabile Prozessführung wäre es somit vorteilhaft, wenn der enthaltene Baryt vor der Verarbeitung ganz bzw. teilweise aus dem Anodenschlamm entfernt werden könnte.

Die systematische Untersuchung der Anodenschlammprobe nach der Methode der aufbereitungstechnischen Merkmalsklassenanalyse hat ergeben, dass Baryt bevorzugt in den gröberen Partikelgrößenklassen vorzufinden ist. Das Ergebnis der Siebklassierung ist in Abb. 3a dargestellt. Wie aus dieser Partikelgrößenverteilung ersichtlich ist, liegt der untersuchte Anodenschlamm durchwegs kleiner $100 \mu \mathrm{m}$ vor. Im rechten Diagramm sind die Ergebnisse der chemischen Analyse ausgewählter Elemente in den verschiedenen Partikelklassen in Form eines Henry-/ReinhardtSchaubildes dargestellt. Aus den beiden Diagrammen können nun beispielhaft folgende Erkenntnisse gewonnen werden: Wird der Trennschnitt bei $25 \mu \mathrm{m}$ gewählt, bedeutet dies, dass das Masseausbringen an Grobgut 15,9\% beträgt und darin immerhin $44 \%$ des Bariuminhaltes der Aufgabe ausgebracht werden können. Gleichzeitig gehen in dieser Fraktion lediglich 3,5\% des Kupferinhaltes, 2,7\% des Bleiinhaltes und jeweils weniger als $1 \%$ des Gold- und Silberinhaltes verloren, weil diese Wertmetalle überwiegend in der Partikelklasse kleiner $25 \mu \mathrm{m}$ vorzufinden sind. Es zeigt sich also auch hier die Möglichkeit, durch einen aufbereitungstechnisch einfachen Klassierschritt einen Sortiereffekt zu erzielen.

\subsection{Schlicker aus der Bleiraffination}

In der Bleimetallurgie ist die stufenweise thermische Bleiraffination das Standardverfahren zur Entfernung von Verunreinigungen wie etwa Kupfer, Arsen, Zinn oder Antimon aus der Bleischmelze. In der ersten Raffinationsstufe, der sogenannten Grobentkupferung, kommt es durch die Reduzierung der Schmelztemperatur auf etwa $380-400^{\circ} \mathrm{C}$ zum Ausseigern von Kupfer und anderen Störmetallen aus der Schmelze. Der sich bildende Schlicker schwimmt aufgrund seiner geringeren Dichte an der Oberfläche der Bleischmelze und wird abgeschöpft. Um die Verarbeitung dieses Schlickers zu vereinfachen, wird mittels eines Rührwerkes Sauerstoff zur Oxidbildung eingebracht. Der Kupfergehalt dieses Schlickers kann zwischen 30 und $45 \%$, der Bleigehalt zwischen 20 und $50 \%$ schwanken. Durch diesen Prozessschritt wird der Kupfergehalt der Bleischmelze auf $0,1-0,5 \%$ gesenkt [8].

In der zweiten Stufe der Raffination werden durch die Zugabe von Natriumnitrat und Natriumhydroxid (dem sogenannten Harris-Prozess, siehe Abb. 4) die Elemente Arsen, Zinn und Antimon aus der Schmelze entfernt.

Zur weiteren Reduktion des Kupfergehaltes der Schmelze wird ihre Temperatur in einer nachfolgenden Prozessstufe bis an ihren Erstarrungspunkt gesenkt. Unter Zugabe von elementarem Schwefel bilden sich CuS- und in weiterer Folge $\mathrm{Cu}_{2} \mathrm{~S}$-Phasen, die abgeschöpft werden. Dieser Schlicker trägt die Bezeichnung Schlicker II und enthält ähnlich
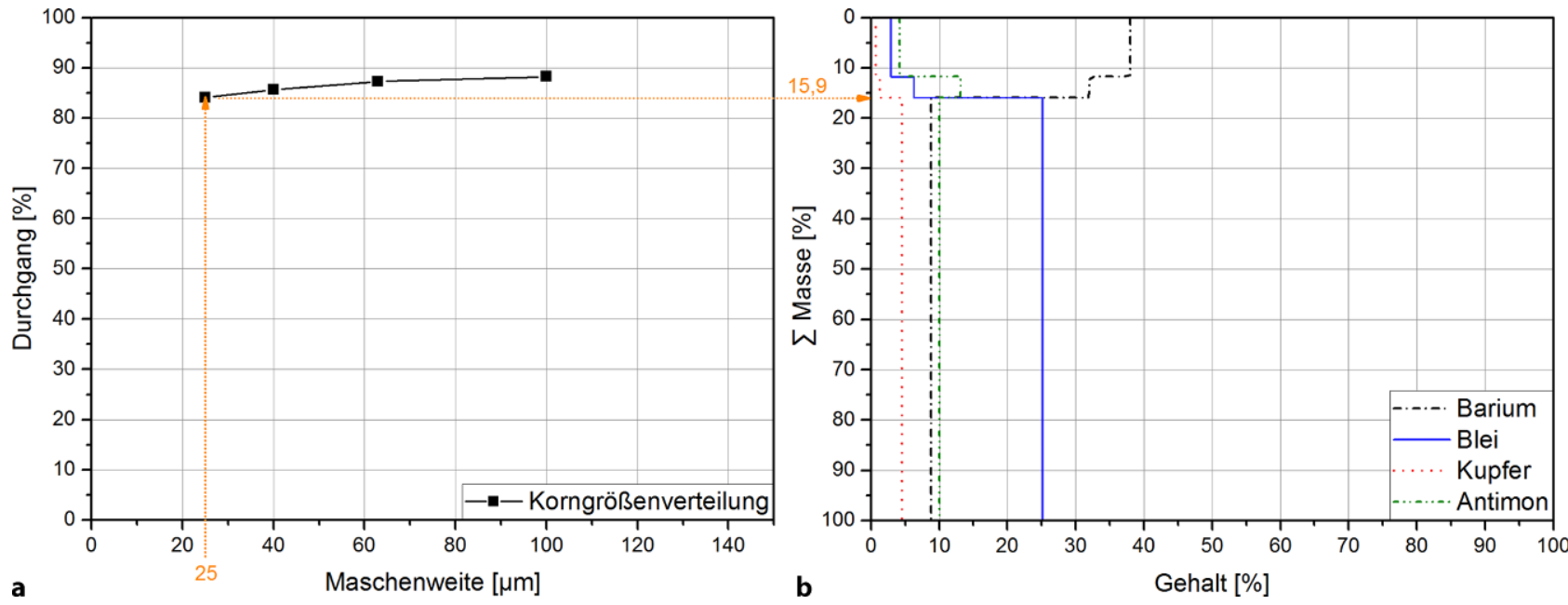

Abb. 3: a Darstellung der Partikelgrößenverteilung des untersuchten Anodenschlammes. b Henry-/Reinhardt-Schaubild für den untersuchten Anodenschlamm 


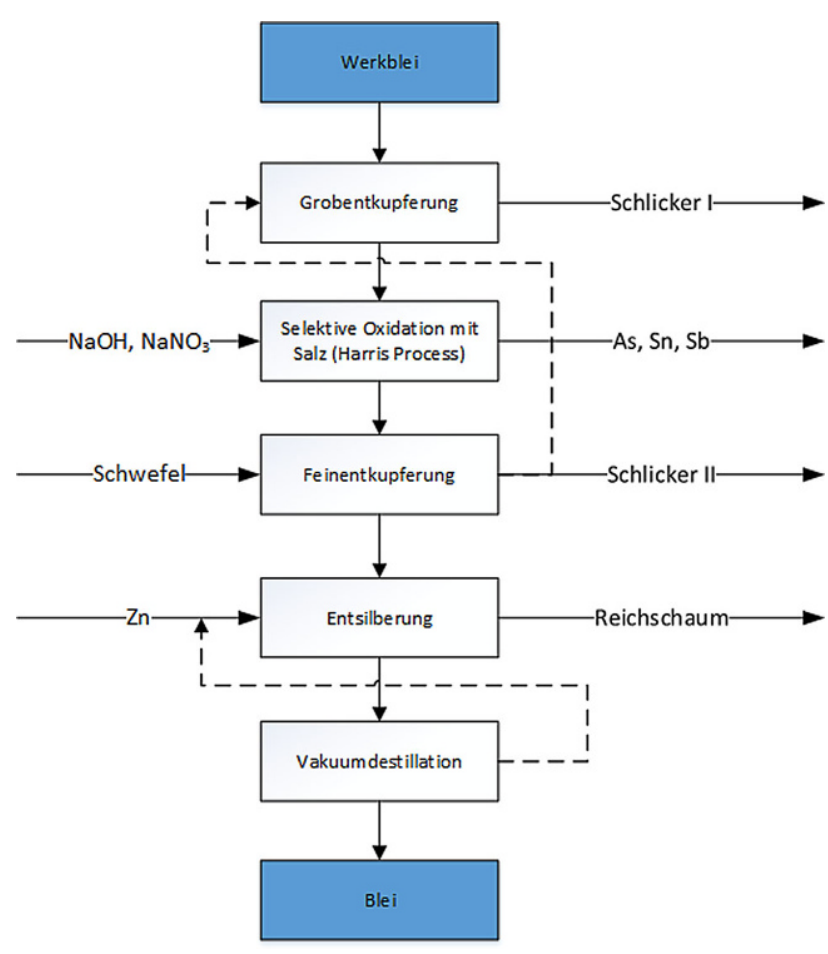

Abb. 4: Vereinfachte Darstellung der thermischen Bleiraffination

wie Schlicker I einen hohen Bleianteil, wobei der Bleigehalt über $50 \%$ liegt.

Beim Abschöpfen des Schlickers werden Anteile der Bleischmelze mitgerissen, was zu einem nicht gewünschten Verlust von Blei und den darin gelösten Edelmetallen führt. Hüttenbetriebe, die in Ergänzung zur Bleilinie auch eine Kupferlinie betreiben, können den solcherart erzeugten Schlicker intern recyceln. Dabei wird aus dem Schlicker mittels eines pyrometallurgischen Prozesses Kupferstein geschmolzen, der in der Kupferlinie weiterverarbeitet werden kann, die bleihaltige Schmelze wird zurück in die Bleiraffination gefahren. Hüttenbetriebe, die nur über eine Bleilinie verfügen und daher keine andere Möglichkeit zur
Aufarbeitung des Schlickers haben, müssen diesen verkaufen. Ein Verkauf bedeutet in diesem Fall einen monetären Wertverlust, da für den Bleianteil und die darin enthaltenen Edelmetalle wie Silber und Gold keine Vergütung erfolgt.

Die Charakterisierung des Schlickers in Hinsicht auf aufbereitungstechnische Aspekte zeigt, dass die vorhandenen Hauptphasen - metallisches Blei und die oxidischen bzw. sulfidischen Kupfer- und Bleiverbindungen wie auch Verunreinigungen wie Eisenoxide - ein unterschiedliches Zerkleinerungsverhalten aufweisen. Durch die Kombination von selektiver Zerkleinerung und nachgeschalteter Klassierung sollte somit eine Anreicherung von metallischer Bleiphase im Groben und oxidischen bzw. sulfidischen Kupfer- und Bleiverbindungen im Feinen möglich sein. Auch Kühn et al. [9] berichten in ihrem Beitrag aus dem Jahr 2009 vom selektiven Zerkleinerungsverhalten von diversen Krätzen und Schlacken. Die Zerkleinerung der Aluminiumsalzschlacke durch eine Rotorprallmühle mit nachfolgender Siebklassierung führt zur Abtrennung des Aluminiums von hydroxidischen und oxidischen Verunreinigen.

Am Lehrstuhl für Aufbereitung und Veredlung wurden dahingehend Versuche mit Schlickerproben aus der Grobentkupferung (Schlicker I) und der Feinentkupferung (Schlicker II) durchgeführt. Die Zerkleinerungsversuche der zwei verschiedenen Schlickerproben erfolgten im Durchlauf mit einer Laborstabmühle. Die Mühle wurde mit $70 \%$ der kritischen Drehzahl betrieben. Unter Variation der Mahldauer wurden mehrere Mahlprodukte der zwei verschiedenen Schlickerarten hergestellt, die in weiterer Folge abermals durch eine aufbereitungstechnische Merkmalklassenanalyse untersucht wurden. Abb. 5 zeigt ausgewählte Ergebnisse aus der Versuchsserie mit den Schlickerproben aus der Grobentkupferung. In Abb. 5a sind die Partikelgrößenverteilungen von drei Mahlprodukten, die einer unterschiedlich langen Mahldauer ausgesetzt waren, dargestellt. In Abb. 5b sind die mittleren Reindichten der verschiedenen Partikelgrößenklassen, bezogen auf das Masseausbringen, dargestellt. Die Partikelgrößenklassen nehmen in dieser Darstellung von oben nach unten ab. Die nachfolgend durchgeführten chemischen Analysen der un-
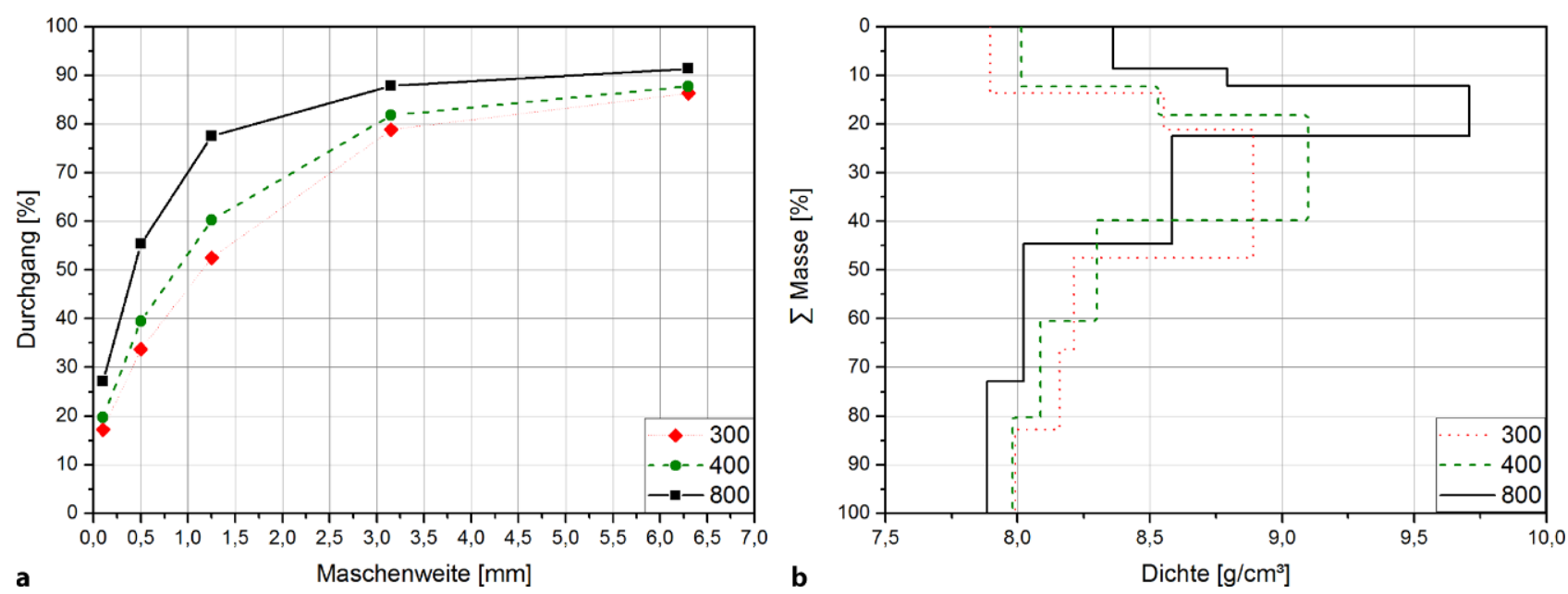

Abb. 5: a Darstellung der Partikelgrößenverteilungen der Zerkleinerungsprodukte unter Variation der Mahldauer (Summe der Umdrehungen). b Darstellung der mittleren Dichten der verschiedenen Partikelklassen in Abhängigkeit von der Mahldauer 

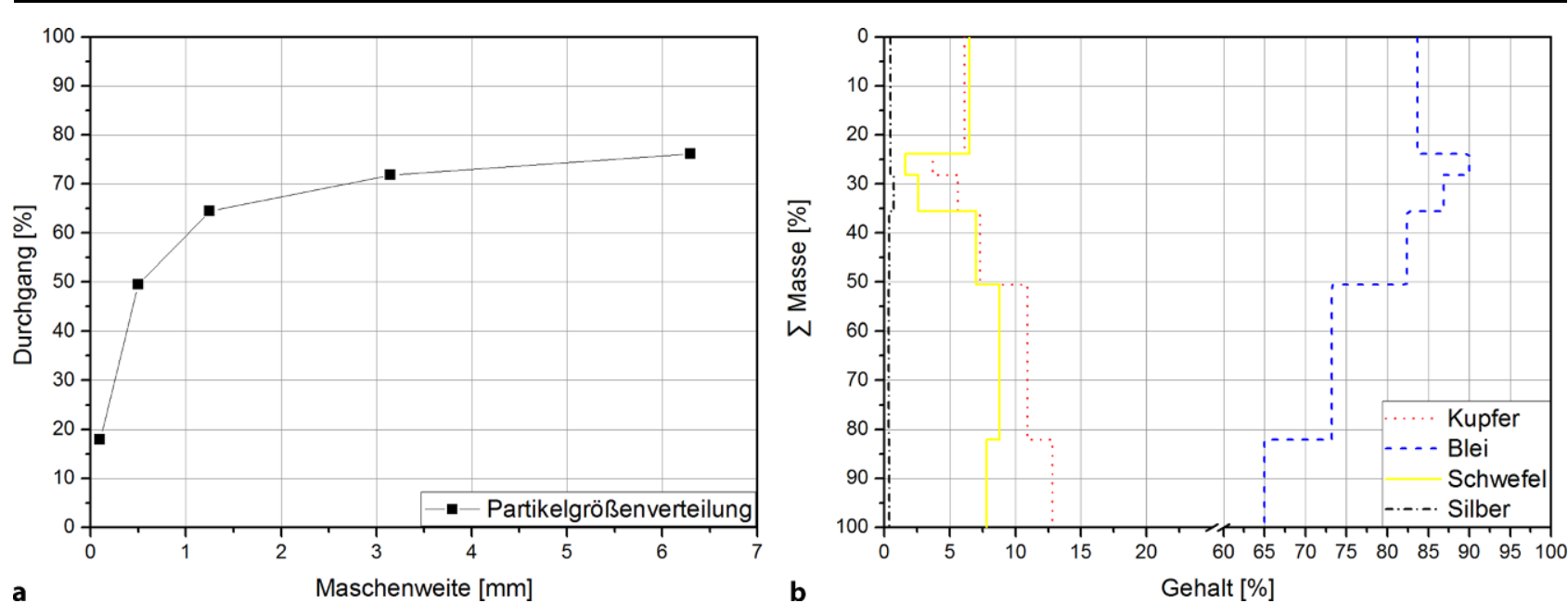

Abb. 6: a Darstellung der Partikelgrößenverteilung des Schlickers aus der Feinentkupferung nach den Zerkleinerungsversuchen. b Dazugehöriges Henry-/Reinhardt-Schaubild mit ausgewählten Elementen. Die Partikelgröße nimmt von oben nach unten ab

terschiedlichen Partikelgrößenklassen haben gezeigt, dass der Bleigehalt in der Partikelgrößenklasse 3,15/1,25 mm bei $79 \%$ liegt, repräsentiert im rechten Diagramm durch die Partikelgrößenklasse mit der mittleren Reindichte von $9,7 \mathrm{~g} / \mathrm{cm}^{3}$. Im Vergleich dazu liegt der mittlere Bleigehalt der untersuchten Schlickerprobe bei lediglich $49 \%$. Durch die Nachreinigung dieser Klasse mittels Handmagnet konnte der Bleigehalt im unmagnetischen Rückstand sogar auf $82 \%$ gehoben werden. Der Kupfergehalt konnte durch die Kombination aus Zerkleinerung in der Stabmühle mit nachfolgender Klassierung und Handmagnetscheidung von $24,5 \%$ in der Aufgabe auf $13,5 \%$ in der genannten Partikelgrößenklasse reduziert werden.

Die Untersuchungen der Schlickerproben aus der Feinentkupferung (Schlicker II) haben ergeben, dass durch die Zerkleinerung in der Stabmühle der Bleigehalt von $77,4 \%$ in der Aufgabe auf bis zu $90 \%$ in der Partikelgrößenklasse $6,3 / 3,15 \mathrm{~mm}$ erhöht werden konnte. In Abb. 6a ist die Partikelgrößenverteilung des Zerkleinerungsproduktes nach einer definierten Mahldauer dargestellt. In Abb. 6b ist die Auswertung der chemischen Analysen der verschiedenen Partikelgrößenklassen in Form eines Henry-/ReinhardtSchaubildes dargestellt. Beim untersuchten Schlicker zeigte sich des Weiteren, dass der Kupfergehalt in den feineren Partikelgrößenklassen ansteigt. So konnte der Kupfergehalt in der Partikelgrößenklasse $<100 \mu \mathrm{m}$ von $8,8 \%$ in der Aufgabe auf $12,8 \%$ erhöht werden.

Die ersten Zerkleinerungsversuche mit den zwei Schlickerproben Schlicker I und Schlicker II haben gezeigt, dass die Zerkleinerungsbeanspruchung der Schlickerproben eine Auswirkung auf die Elementverteilung in den betrachteten Partikelgrößenklassen der verschiedenen Zerkleinerungsprodukte hat. Tendenziell ist ersichtlich, dass sich Blei in den gröberen Partikelklassen anreichert, was sich auf das duktile Verhalten der metallischen Bleiphasen zurückführen lässt.

\section{Schlussbemerkungen}

Aus der Aufbereitung von Industriemineralen sind Anwendungsfälle bekannt, wo durch die Verwendung eines Klassierschrittes ein Sortiereffekt erzielt werden kann. In der Nichteisenmetallurgie gibt es einige Beispiele, in denen eine Klassierung der Zwischenprodukte neue Wege der Aufarbeitung dieser Stoffe bietet. Etwa bei der Aufarbeitung von Flugstäuben, wo die Klassierung die Möglichkeit bietet, chemisch-physikalisch gebildete Anteile von mechanisch mitgerissenen Anteilen abzutrennen. In der Aufarbeitung von Anodenschlämmen aus der Kupferelektrolyse ermöglicht die Klassierung die teilweise Abtrennung der beim Anodengießen eingesetzten Schlichte, die störend auf nachfolgende Prozesse wirkt. In der Bleiraffination wiederum ermöglicht die Kombination aus Zerkleinerung und anschließender Klassierung, die Verluste an metallischem Blei und somit auch an den darin enthaltenen EdelmetalIen in den beiden Schlickerprodukten zu reduzieren. Auf Basis der derzeitigen Ergebnisse der Untersuchungen erscheint eine Fortsetzung der Untersuchungen für weitere metallurgische Prozesse sinnvoll.

Acknowledgements. Open access funding provided by Montanuniversität Leoben.

Open Access Dieser Artikel wird unter der Creative Commons Namensnennung 4.0 International Lizenz (http://creativecommons.org/licenses/ by/4.0/deed.de) veröffentlicht, welche die Nutzung, Vervielfältigung,

Bearbeitung, Verbreitung und Wiedergabe in jeglichem Medium und Format erlaubt, sofern Sie den/die ursprünglichen Autor(en) und die Quelle ordnungsgemäß nennen, einen Link zur Creative Commons Lizenz beifügen und angeben, ob Änderungen vorgenommen wurden. 


\section{Literatur}

1. Schubert, H.: Aufbereitung fester mineralischer Rohstoffe, Bd. 1. Leipzig: VEB Deutscher Verlag für Grundstoffindustrie, 1975

2. Kaltenreiner $E$. Entwicklung und Stand der Kaolinaufbereitung in Österreich, in: Flachberger, H.; Waldl, G.; Kolb, H.; Haslinger, S.; Bauer-Vasko, C. (Hrsg.): Aufbereitung in Österreich, Leoben: Eigenverlag, 2011, S. 25-29

3. Samuelsson, C.; Carlsson, G.: Characterization of copper smelter dusts, CIM Bulletin 94(1051), (2001), S. 111-115

4. Schlesinger, M.E.; King, M.J.; Sole, K.C.; Davenport, W.G. (ed.): Extractive Metallurgy of Copper, 5. ed., Amsterdam (etc.): Elsevier, 2011

5. Öfner, W.: Anwendung der Merkmalsklassenanalyse auf feinkörnige Reststoffe aus der Eisen- und Stahlindustrie, BHM Berg- und Hüttenmännische Monatshefte, 158 (2013), H. 6, S. 244-250
6. Rumpold, R.; Antrekowitsch, J.: Removal of Halogens in Heavy Metal Containing Flue Dust from the Secondary Copper Production, Proceedings of EMC 2013, European Metallurgical Conference. June 23-26, 2013, Weimar, Germany. GDMB Verl., Clausthal-Zellerfeld, S. $933-938$

7. Wenzel, C.: Structure and Casting Technology of Anodes in Copper Metallurgy. Diss., Leoben, Montanuniversität, Lehrstuhl für Nichteisenmetallurgie, 2008

8. Sinclair, R.J.: The extractive metallurgy of lead: Australasian Institute of Mining and Metallurgy Spectrum series Volume Number 15, Carlton Victoria: AusIMM the Australasian Inst. of Mining and Metallurgy, 2009

9. Kühn, C.; Schicht, E.: Prallbeanspruchung für selektive Zerkleinerung, AT Mineral processing, 6 (2009), S. 62-69 